\title{
Minireview
}

\section{The absolute pitch mind continues to reveal itself}

\author{
Patrick Bermudez ${ }^{* \dagger}$ and Robert J Zatorre*†
}

\begin{abstract}
Addresses: *Montreal Neurological Institute, McGill University, 3801 University Street, Montréal, Québec, H3A 2B4 Canada. tBRAMS Laboratory, 1420 boul. Mont Royal, Montréal, Québec, H3C 3J7 Canada.
\end{abstract}

Correspondence: Patrick Bermudez. Email: patrick@bic.mni.mcgill.ca

\begin{abstract}
Absolute pitch has proved useful as an alternative perspective from which to investigate various cognitive faculties. A new functional magnetic resonance imaging study published recently in $B M C$ Neuroscience adds new data to the ongoing debate concerning the neural underpinnings of this unusual ability.
\end{abstract}

Root, fifth, sixth, flatted seventh, octave, ninth, flatted seventh, octave, ninth, octave. When played with the right rhythm and attitude, these musical intervals will be recognized by many people as the main thematic refrain from So What, by Miles Davis. We are able to discern this because the pattern of intervallic relationships (of both pitch and time) forms the melody, no matter the starting pitch. If given the information that the root note is ' $\mathrm{D}$ ', any trained musician will be able to compute the rest of the note names by identifying the intervallic distances between each note of the melody and the root (or any other note that has been determined), yielding D, A, B, C, D, E, C, D, E, D. This ability is referred to as relative pitch. A small minority of musicians, however, can identify all the notes 'absolutely', each in isolation and without reference to any other note. In other words, they possess some sort of internal template, a stable representation of pitches to which they can compare the incoming signal and subsequently identify the notes by name, by sounding them on an instrument, or other responses. This is referred to as perfect or absolute pitch (AP). A comprehensive review of the factors influencing the likelihood of acquiring AP is far beyond the scope of this brief commentary, although the two most frequently mentioned are early musical training (particularly during an early developmental phase during which there is relatively preserved affinity for absolute information), and a predisposing biology, the nature of which is still largely speculated. As we will see shortly, a brain functional magnetic resonance imaging study comparing musicians with and without AP published recently in BMC Neuroscience by Schulze and colleagues [1] now fits another piece to the puzzle.

\section{Scientific attraction}

As scientists, why should we care about what seems to be a musical quirk? Most would agree that where there is a difference in behavior, there must be a neural correlate to this difference, perhaps qualitative or merely quantitative. The necessary cognitive components required to execute a typical absolute pitch task (which can be easily operationalized for musicians) are such that AP becomes a model with which one can explore perceptual and mnemonic faculties and further the larger endeavor of mapping functional specialization in the brain. Add to that the fact that not all who are exposed to equivalent musical tuition develop clear forms of AP and you have the motivation for a number of questions about the interaction between genetics and environment. This possibility has broader appeal still if one considers that absolute pitch is perhaps not perfectly cognitively self-encapsulated and uniquely musical (as it has usually been described). What if it were a musical-domain manifestation of more general features of cognition? The most typical report from possessors of AP is that they acquired the ability effortlessly and incidentally, even assuming that everyone else was hearing what they heard until they were told otherwise. This is consistent with the suggestion that one of the crucial factors in determining whether or not one develops AP may be a global cognitive predilection that affects the way in which some people distil and process the information impinging upon their senses. Indeed, recent evidence has shown AP proficiency to be correlated with field-independent and sequential processing ([2] and references therein). Ultimately, it is likely that these cognitive inclinations interact with developmental, tuitional and genetic contributions in a propitious confluence of factors, each with its weighted contribution to the emergence of a variety of AP proficiencies.

It follows from this perspective on AP that the genetic/ biological contributions (presumably existing to varying degrees throughout the larger population) give rise to a number of interacting features, including the ability to retain isolated information, a cognitive predilection towards field-independent/narrow attention processing [3], and perhaps an auditory system capable of rendering unusually salient and rich descriptions of the stimuli [4]. The implication, of course, is that we may not be looking for an AP gene per se but rather a constellation of genes influencing these broad facets of cognition. Although familial aggregation studies have suggested that there is likely shared biology 


\section{Right hemisphere}

\section{Left hemisphere}

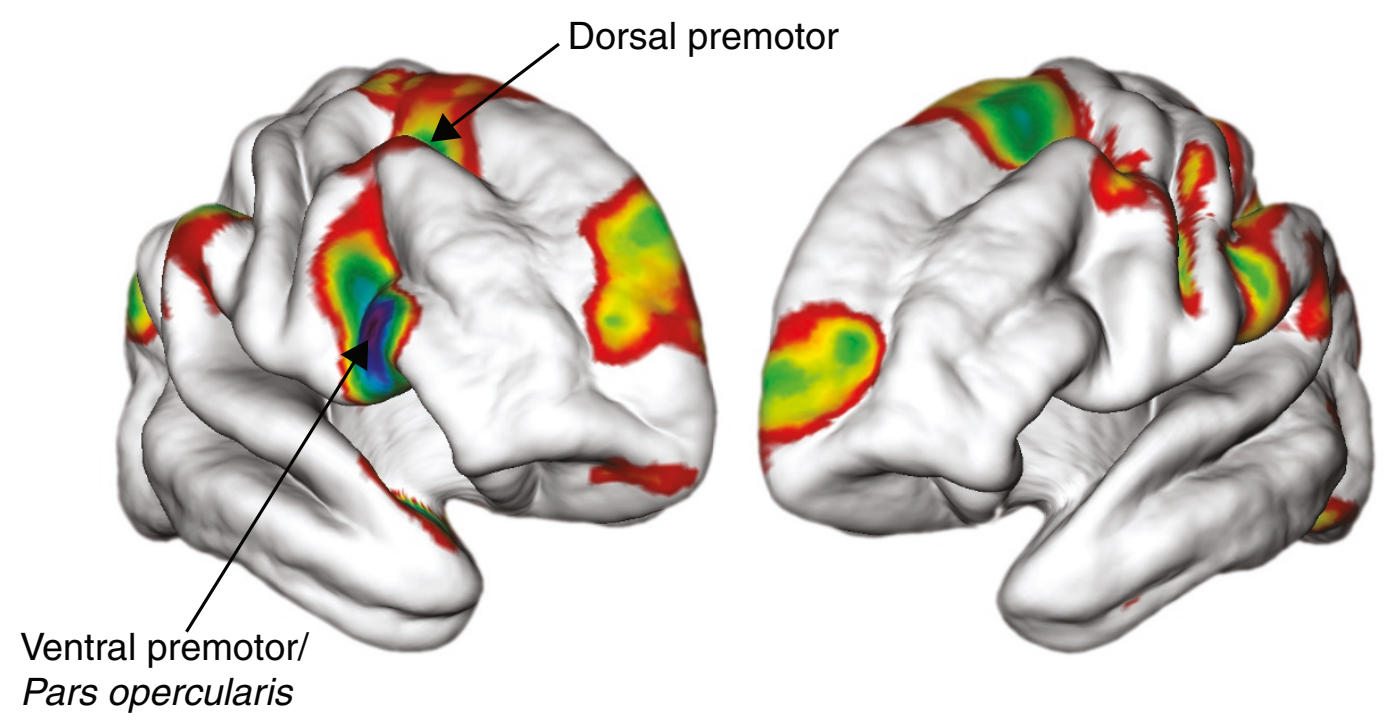

Figure 1

Differences in cortical thickness between musicians with and without absolute pitch in areas of the frontal lobe. Colored regions indicate statistically significant differences in cortical thickness between the 12 strongest and 12 weakest performers on a test of absolute pitch (AP). Adapted from Bermudez et al. [11].

and environment among relatives, which interact to favorably dispose individuals to the acquisition of AP, there is, at the moment, little evidence to support a single-gene origin for a perfectly self-encapsulated expression of AP (see [5] for a brief overview). Only when we combine further genetic, behavioral and neuroscientific knowledge will we be in a position to posit firmer mechanisms.

\section{Neural correlates}

Now, what of the brain in all of this? The last quarter century or so of research into AP has given us the first indications that possessors may show neuroanatomical and functional correlates of their unusual ability [5,6]. For instance, in 1984 Klein and colleagues showed a diminished amplitude of the $\mathrm{P}_{3} 00$ evoked electrical potential (taken to be an index of the refreshing of working memory context) in response to the infrequent stimulus in an oddball paradigm. It was therefore proposed that possessors of AP had less need for tonal working memory compared with non-possessors, given their ability to recode the stimulus into a categorical descriptor (for example, B flat). A pair of studies by our group $[7,8]$ showed how the posterior dorsolateral frontal cortex is implicated in the conditional associative memory required for the correct matching of notes to note names by possessors of AP.

The first suggestion of distinguishing anatomy came by way of an in vivo study by Schlaug et al. [9] showing that the typical leftward hemispheric asymmetry of the surface area of the planum temporale (first shown in 1968 by Geschwind and Levitsky in post-mortem brains) was exaggerated in the brains of AP musicians compared to their non-AP musician cohort. In 2001, Keenan et al. [10] showed how a similar effect seemed to be driven by a smaller right rather than larger left surface area. Since the work of Schlaug et al. [9], most efforts at examining AP cerebral function and morphology have targeted working memory and the planum temporale, although more recently, one of us (PB) and colleagues [11] revealed differences in cortical thickness between musicians with and without absolute pitch in dorsal premotor, ventral premotor and par opercularis areas of the frontal lobe (Figure 1). These first findings outside secondary auditory areas propounded the idea that AP possessors show a profile of anatomical differences that is different from that of either other musicians or non-musicians.

The recent work by Schulze, Gaab and Schlaug reported in $B M C$ Neuroscience [1] uses functional magnetic resonance imaging to investigate differences between $\mathrm{AP}$ and non-AP musicians with a pitch memory task that required subjects to retain a series of tones and later identify whether the last or second-to-last tone was the same as or different from the first. The authors' experimental design allowed inspection of early activity, occurring o to 3 seconds after the end of stimulus presentation, presumably reflecting perceptual 


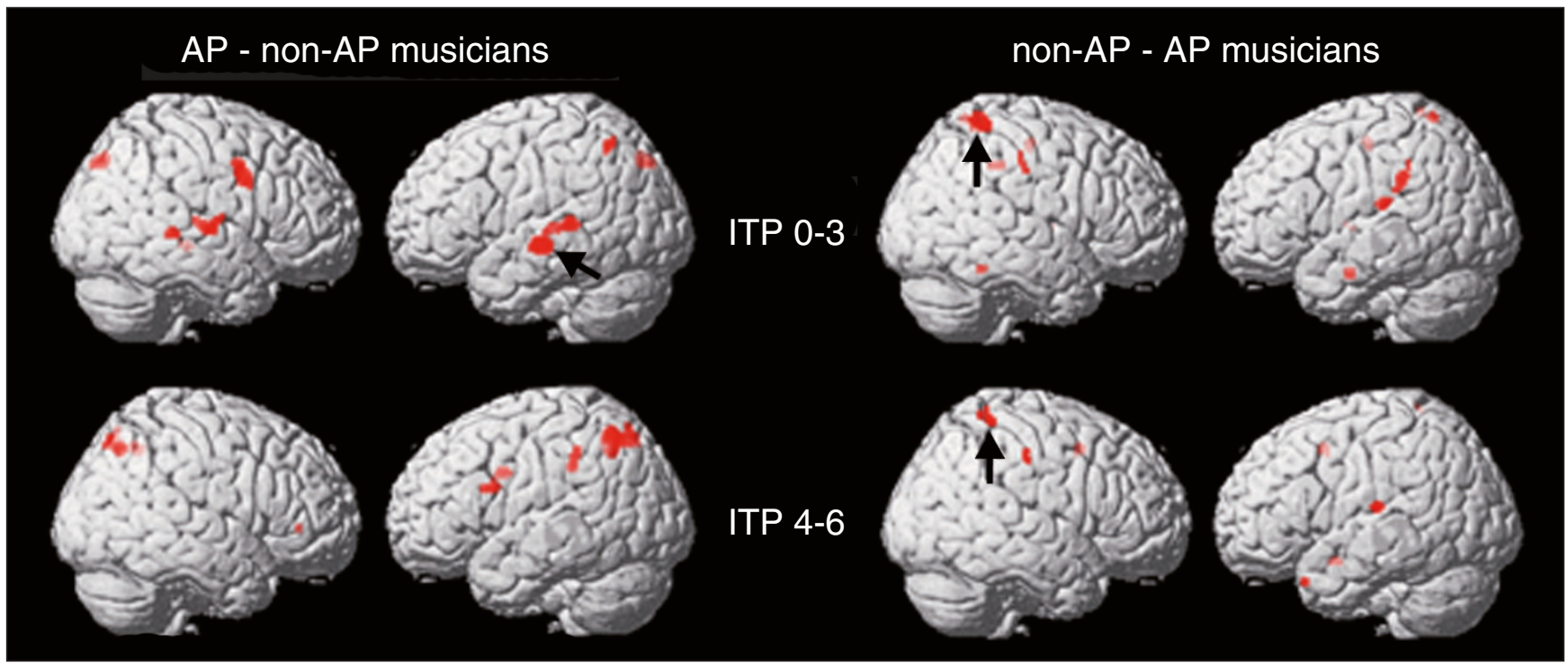

Figure 2

Differences in early and late activity between absolute pitch (AP) and non-AP musicians. AP possessors showed greater activity in the left superior temporal sulcus during the early scanning period (imaging time points (ITPs) at 0 to $3 \mathrm{~s}$ post-stimulus) and non-possessors showed greater activity in right parietal areas during both scanning periods (ITPs at 4 to 6 s post-stimulus).

encoding, and later activity from 4 to 6 seconds poststimulus, thought to reflect post-encoding functions such as working memory. Schulze et al. found largely overlapping activity between the groups, though, when contrasting the AP and non-AP musicians directly, AP musicians showed greater activity in the left superior temporal sulcus (STS) during the early scanning period and non-AP musicians showed greater activity in right parietal areas during both scanning periods (Figure 2).

The authors interpret these effects as reflecting perceptual and encoding differences among the AP subjects, drawing a plausible link to literature demonstrating the importance of the STS in the identification and categorization of sounds, and increased reliance on working memory for the non-AP subjects, as expected from previous work showing the likely recoding of notes into less working-memory intensive forms by subjects possessing AP. The parietal activity may also reflect a type of operation similar to that necessary for relative pitch judgments and melodic transposition [12]. These findings sum themselves to and extend a literature suggesting differences in encoding of notes and a consequent difference in working-memory requirements between AP and non-AP musicians.

\section{The search continues}

Focus on both the function and morphology of the temporal region has been fruitful, although much work remains to be done to clarify the full significance of its role in AP. The findings of Schulze et al. [1] make a significant contribution to the emerging picture and give us confidence that this region warrants further study. As mentioned above, we now have indications that the morphology of some areas other than those involved in higher auditory processing may be correlated with AP ability. If we discover more correlations between AP ability and other facets of cognition, and more widespread anatomical findings in areas that subserve functions extending far beyond the requisites of $\mathrm{AP}$, we will be increasingly compelled to consider the possibility that some forms of AP may, in part, be manifestations in the musical domain of more comprehensive elements of cognition. This view of $\mathrm{AP}$ as a multifactorial emergent consequence that might have correlates in other spheres of cognitive experience (and their cerebral substrates) has been underexplored in the AP literature, despite its having certain parsimonious advantages. Furthermore, it enriches the subject matter and what it promises to teach us. We may also yet discover that the very strongest forms of AP are supported by discretely unique biology that acts qualitatively differently from that of most of the population. Regardless of what may be the eventual Truth of the matter, AP continues to serve as an interesting model for neuroscientific discovery.

\section{Acknowledgements}

We gratefully acknowledge the support of the Canadian Institutes of Health Research and the International Foundation for Music Research.

\section{References}

1. Schulze K, Gaab N, Schlaug G: Perceiving pitch absolutely: comparing absolute and relative pitch possessors in a pitch memory task. BMC Neurosci 2009, 10:106. 
2. Moreno Sala M, Costa-Giomi E: The relationship between selected cognitive abilities and young children's pitch identification. Psychol Music 2009, in press.

3. Chin CS: The development of absolute pitch: a theory concerning the roles of music training at an early developmental age and individual cognitive style. Psychol Music 2003, 31:155-171.

4. Ross DA, Gore JC, Marks LE: Absolute pitch: music and beyond. Epilepsy Behav 2005, 7:578-601.

5. Zatorre RJ: Absolute pitch: a paradigm for understanding the influence of genes and development on cognitive function. Nat Neurosci 2003, 6:692-695.

6. Levitin DJ, Rogers SE: Absolute pitch: perception, coding, and controversies. Trends Cogn Sci 2005, 9:26-33.

7. Bermudez P, Zatorre RJ: Conditional associative memory for musical stimuli in nonmusicians: implications for absolute pitch. J Neurosci 2005, 25:7718-7723.

8. Zatorre RJ, Perry DW, Beckett CA, Westbury CF, Evans AC: Functional anatomy of musical processing in listeners with absolute pitch and relative pitch. Proc Natl Acad Sci USA 1998, 95:3172-3177.
9. Schlaug $\mathrm{G}$, Jancke $\mathrm{L}$, Huang $\mathrm{Y}$, Steinmetz $\mathrm{H}$ : In vivo evidence of structural brain asymmetry in musicians. Science 1995 267:699-701.

10. Keenan JP, Thangaraj V, Halpern AR, Schlaug G: Absolute pitch and planum temporale. Neuroimage 2001, 14:14021408.

11. Bermudez P, Lerch JP, Evans AC, Zatorre RJ: Neuroanatomical correlates of musicianship as revealed by cortical thickness and voxel-based morphometry. Cereb Cortex 2009, 19:1583-1596.

12. Foster $\mathrm{N}$, Zatorre $\mathrm{R}$ : A role for the intraparietal sulcus in transforming musical pitch information. Cereb Cortex 2009, in press.

Published: 27 August 2009

doi:10.1186/jbiol171

(c) 2009 BioMed Central Ltd 